\title{
O Papel da Testosterona na Melhoria do Desejo Sexual da Mulher Pós-Menopáusica: Uma Revisão Baseada na Evidência
}

\section{The Role of Testosterone in The Improvement of Sexual Desire in Postmenopausal Women: An Evidence-Based Clinical Review}

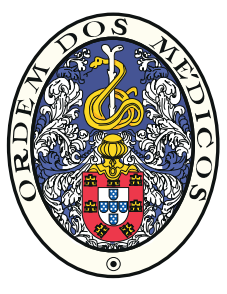

\author{
Maria GOUVEIA $\triangle^{1}$, Raquel SANCHES ${ }^{1}$, Sara ANDRADE ${ }^{2}$, Sara CARMONA ${ }^{1}$, Carolina FERREIRA ${ }^{1}$ \\ Acta Med Port 2018 Nov;31(11):680-690 • https://doi.org/10.20344/amp.9277
}

\section{RESUMO}

Introdução: A disfunção sexual feminina é um problema comum que atinge mais de 1/3 das mulheres em alguma fase da vida. $O$ objetivo deste trabalho é rever a evidência existente acerca da eficácia da testosterona na disfunção sexual, em particular na melhoria do desejo, em mulheres pós-menopáusicas.

Material e Métodos: Pesquisa nas bases de dados National Guidelines Clearinghouse, Guidelines Finder, The Cochrane Library e MEDLINE/PubMed, de normas de orientação clínica, revisões sistemáticas, meta-análises e ensaios clínicos aleatorizados e controlados, publicados entre janeiro de 2005 e fevereiro de 2017, utilizando os termos MeSH 'testosterone', 'androgens', 'libido', 'sexual dysfunctions' e 'menopause'.

Resultados: Foram incluídos 11 artigos de uma pesquisa inicial de 506: três normas de orientação clínica, uma revisão sistemática com meta-análise e sete ensaios clínicos aleatorizados e controlados. Os artigos selecionados mostraram eficácia da terapêutica com testosterona, em monoterapia ou em associação com outras hormonas, na melhoria global da função sexual e na melhoria do desejo sexual em mulheres na pós-menopausa. Nenhum dos estudos evidenciou alterações das enzimas hepáticas ou efeitos adversos graves.

Discussão: Os estudos incluídos tinham amostras de pequenas dimensões e período de follow-up curto, o que impossibilitou a avaliação dos efeitos a longo prazo do tratamento com testosterona.

Conclusão: A terapêutica com testosterona tem benefício, a curto prazo, na melhoria das queixas de disfunção sexual em mulheres pós-menopáusicas, em particular na melhoria do desejo. Contudo, são necessários estudos com amostras de maior dimensão e período de follow-up mais longo, de modo a avaliar a sua efetividade e segurança a longo prazo.

Palavras-chave: Androgénios; Disfunções Sexuais Psicogénicas/tratamento; Libido; Pós-Menopausa; Testosterona

\section{ABSTRACT}

Introduction: Female sexual dysfunction is a common problem, affecting more than $1 / 3$ of women during their lives. The aim of this review is to review the evidence for the effectiveness of testosterone in sexual dysfunction in postmenopausal women, particularly in the improvement of sexual desire.

Material and Methods: The authors searched in international databases National Guidelines Clearinghouse, Guidelines Finder, Cochrane Library and MEDLINE/PubMed, for guidelines, systematic reviews, meta-analysis and randomized controlled trials, published between January 2005 and February 2017, using the MeSH terms 'testosterone', 'androgens', 'libido', 'sexual dysfunctions' and 'menopause'.

Results: From a pool of 506 articles, 11 were selected: three guidelines, one systematic review with meta-analysis and seven randomized controlled trials. The selected articles showed testosterone's efficacy on global sexual function and improvement of sexual desire in postmenopausal women, when both are used in monotherapy or in association with other hormones. No study showed changes in hepatic enzymes or serious adverse effects.

Discussion: The small sample size and short follow-up used in the included studies limits the ability to assess testosterone's long-term benefits and effects.

Conclusion: At short-term, testosterone seems to improve sexual function in postmenopausal women, particularly sexual desire. Nevertheless, more studies with larger sample size and longer follow-up are needed to understand its long-term safety and effectiveness.

Keywords: Androgens; Libido; Postmenopause; Sexual Dysfunctions, Psychological/drug therapy; Testosterone

\section{INTRODUÇÃO}

A disfunção sexual feminina (DSF) é um problema comum que atinge mais de $1 / 3$ das mulheres ao longo da sua vida e que tem um impacto negativo no humor, autoestima e qualidade de vida. ${ }^{1-4}$ Está associada à menopausa e ao envelhecimento, sendo que a maioria das mulheres pós-menopáusicas sexualmente ativas refere pelo menos uma dificuldade a nível da relação sexual, como a redução do desejo. ${ }^{1}$

A classificação norte-americana do Manual de Diagnóstico e Estatística das Perturbações Mentais, quarta edição, na sua edição de texto revisto (DSM-IV-TR), determina quatro categorias de DSF: desejo sexual hipoativo (DSH), perturbação da excitação, perturbação do orgasmo e perturbação sexual dolorosa. A presença de sofrimento é

1. Unidade de Saúde Familiar S. Julião. Agrupamento de Centros de Saúde de Lisboa Ocidental e Oeiras. Lisboa. Portugal.

2. Unidade de Saúde Familiar Conde de Oeiras. Agrupamento de Centros de Saúde de Lisboa Ocidental e Oeiras. Lisboa. Portugal.

$\triangle$ Autor correspondente: Maria Gouveia. maria.folque.gouveia@gmail.com

Recebido: 07 de junho de 2017 - Aceite: 25 de agosto de 2018 | Copyright $\odot$ Ordem dos Médicos 2018 
fundamental para estes problemas serem diagnosticados como disfunções. ${ }^{5,6}$ Considerando que desejo e excitação são difíceis de distinguir, o Manual de Diagnóstico e Estatística das Perturbações Mentais, quinta edição (DSM 5) reúne o diagnóstico de DSH com o de perturbação da excitação, sendo esta condição classificada como perturbação do interesse/excitação sexual feminina. ${ }^{4,7}$

O DSH é a DSF mais frequente relacionando-se com a idade (pico entre 45 - 64 anos) e a menopausa. ${ }^{3,8}$ Afeta $10 \%-12 \%$ das mulheres, podendo atingir $26 \%$ nas muIheres submetidas a menopausa cirúrgica (por norma secundária à realização de histerectomia, associada a ooforectomia bilateral). ${ }^{3} \mathrm{DSH}$ define-se como a ausência e/ou diminuição persistente ou recorrente de: fantasias e pensamentos sexuais/eróticos, interesse e recetividade para a atividade sexual, resposta a estímulos sexuais, e prazer e sensibilidade durante a atividade sexual. Estes sintomas devem estar presentes há pelo menos seis meses e não podem ser explicados por doença mental, medicamentos ou outra condição médica, nem ser consequência de uma relação conflituosa (em que há, por exemplo, violência do/a parceiro/a). ${ }^{3,5}$

Uma constelação de fatores biológicos, relacionais, psicológicos e socioculturais, interrelacionam-se e determinam o desejo sexual, pelo que a etiologia desta disfunção é geralmente multifatorial. $^{2,5,8}$

$\mathrm{Na}$ menopausa, a diminuição do desejo sexual pode dever-se à redução dos estrogénios e ao declínio da produção de androgénios pelos ovários. A reposição em estrogénios é efetiva no tratamento da secura vaginal e dispareunia, mas no que diz respeito ao desejo sexual, a menos que a sua redução seja consequência da dor ou perda de sensibilidade genital, o seu efeito não foi demonstrado. ${ }^{4}$

À medida que a mulher envelhece, a produção de androgénios pelas glândulas suprarrenais decresce, o que leva a um declínio progressivo da testosterona sérica resultante da conversão periférica. Na pós-menopausa, mantém-se a produção ovárica de testosterona, mas ocorre uma redução de $50 \%$ na produção de androstenediona, contribuindo para os níveis mais baixos de testosterona sérica. ${ }^{2}$ Isto explica que a mulher aos 20 anos tenha concentrações máximas de androgénios e que aos 50 estas se reduzam para metade..$^{2-4}$

A relação entre os androgénios e o desejo sexual foi estabelecida há 75 anos e desde 1980 que tem sido alvo de estudos. ${ }^{4}$ Shifren et a/ ${ }^{9}$ publicaram em 2000 um ensaio clínico aleatorizado e controlado que incluiu 75 mulheres que receberam, durante 12 semanas, estrogénios conjugados associados a placebo ou a 150 ou $300 \mu \mathrm{g}$ microgramas de testosterona transdérmica. Este estudo mostrou uma melhoria significativa de vários parâmetros da função sexual, incluindo o desejo, e do bem-estar psicológico, com a dose mais alta de testosterona. De igual modo, várias revisões da literatura mostraram um efeito benéfico da testosterona na melhoria da função sexual, em particular do desejo, em mulheres pós-menopáusicas. ${ }^{2,4}$ Hubayter et $a l,{ }^{2}$ por exemplo, conduziram uma revisão da literatu- ra, publicada em 2008, cujos resultados mostraram uma melhoria da função sexual com a terapêutica com testosterona em mulheres pós-menopáusicas com DSH, sobretudo após ooforectomia. Noutra revisão da literatura, publicada em $2013,{ }^{4}$ é também referido um efeito benéfico da testosterona transdérmica, isolada ou associada a estrogénios, na melhoria da satisfação sexual e do desejo em mulheres pós-menopáusicas.

Além disso, esta hormona aumenta o fluxo de sangue vaginal e a integridade deste tecido. Por outro lado, níveis baixos de testosterona foram associados a fadiga persistente, diminuição do bem-estar físico e menor prazer/desejo sexual. ${ }^{2-4}$ Apesar de sugerido que as flutuações nos níveis de testosterona na mulher podem ser responsáveis pela redução da líbido, não se estabeleceu, ainda, uma associação clara entre esta hormona e a função sexual, ${ }^{2,4}$ desconhecendo-se o limite sérico mínimo que se correlaciona com a DSF. ${ }^{5}$

Os potenciais riscos da terapêutica com androgénios incluem: hirsutismo, acne, virilização da voz e alteração da função hepática e do perfil lipídico. Como os androgénios também são aromatizados em estrogénios, o risco de doença cardiovascular (CV) e de cancro da mama também é possível na terapêutica com testosterona. ${ }^{8}$

Os estudos de segurança sobre a utilização de androgénios na mulher são escassos e desconhecem-se os seus efeitos a longo prazo. ${ }^{3,4,8}$ Atualmente, os produtos utilizados estão apenas aprovados para homens e são prescritos off-label para mulheres. Existem diversas vias de administração no mercado internacional (testosterona oral, transdérmica, subcutânea, sublingual e intramuscular). ${ }^{2}$ Em Portugal, a testosterona é comercializada apenas em gel para administração por via transdérmica e em solução injetável por via intramuscular. ${ }^{10}$

As farmácias também disponibilizam manipulados, cuja qualidade, efetividade e efeitos adversos são de mais difícil controlo, uma vez que não são estudados. ${ }^{2}$

O objetivo deste trabalho é rever a evidência existente acerca da eficácia da testosterona na disfunção sexual, em particular na melhoria do desejo, em mulheres pós-menopáusicas.

\section{MATERIAL E MÉTODOS}

Recorrendo às bases de dados da National Guidelines Clearinghouse, Guidelines Finder, The Cochrane Library e MEDLINE/PubMed, realizou-se uma pesquisa de normas de orientação clínica (NOC), revisões sistemáticas (RS), meta-análises (MA) e ensaios clínicos aleatorizados e controlados (ECAC) publicados entre janeiro de 2005 e fevereiro de 2017, na língua inglesa. Foram utilizados os termos MeSH 'testosterone', 'androgens', 'libido', 'sexual dysfunctions' e 'menopause' com a seguinte estratégia de pesquisa "(testosterone OR androgens) AND (libido OR sexual dysfunctions) AND (menopause)". Fez-se ainda pesquisa manual de referências bibliográficas para identificar estudos relevantes que pudessem não ter sido incluídos na pesquisa nas bases de dados. 
Os critérios utilizados para a inclusão dos artigos foram: população constituída por mulheres na pós-menopausa (fisiológica ou cirúrgica) com queixas de disfunção sexual, quantificada em questionário; intervenção com testosterona por qualquer via de administração, isoladamente ou em combinação com estrogénio e/ou progesterona; comparação com outra terapêutica hormonal (estrogénio) ou não hormonal (lubrificante) ou placebo; outcome constituído por melhoria das queixas de disfunção sexual após seguimento (individualizando-se resultados na parte do desejo), quantificada através de questionário. Definiram-se como critérios de exclusão: homens; mulheres com história de neoplasia maligna uro-ginecológica; mulheres a fazer terapêutica ou com doença subjacente que possa contribuir/causar disfunção sexual e estudos que abordassem outros parâmetros de disfunção sexual mas não incluíssem o desejo. O desejo e a excitação sexual, nos resultados, foram apresentados de forma independente, tal como é definido pela DSM-IV. ${ }^{6}$

Para estratificar o nível de evidência (NE) dos estudos e a força de recomendação (FR) foi utilizada a Strenght of
Recommendation Taxonomy (SORT) da American Academy of Family Physicians. ${ }^{11}$

A seleção dos artigos para revisão foi feita em duplicado por dois autores e em caso de discordância foi discutida pelo par a inclusão/exclusão com uma taxa de concordância final de $100 \%$. Realizou-se a leitura integral dos artigos selecionados e a avaliação de qualidade e atribuição de nível de evidência dos estudos foi discutida por todas as autoras.

\section{RESULTADOS}

Da pesquisa efetuada resultou um total de 506 artigos, 505 encontrados através de pesquisa em base de dados e um artigo encontrado por pesquisa manual. Destes, apenas 11 cumpriam os critérios de inclusão: três NOC, uma RS com MA e sete ECAC. A Fig. 1 representa o fluxograma de seleção dos estudos.

\section{Normas de orientação clínica}

As recomendações das normas de orientação clínica estão resumidas na Tabela 1.

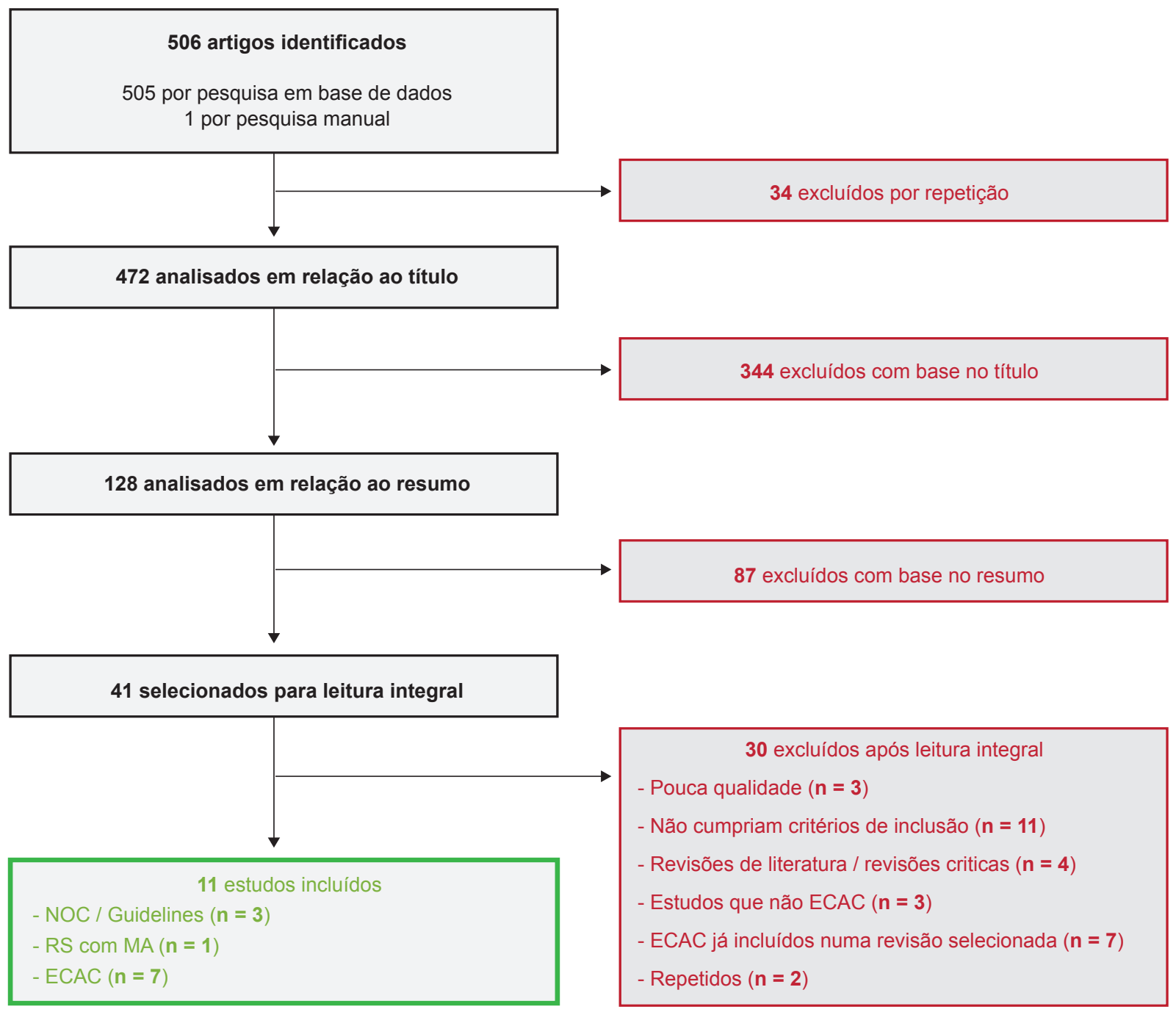

Figura 1 - Fluxograma de seleção dos estudos 
Tabela 1 - Resumo das recomendações das normas de orientação clínica

\begin{tabular}{|c|c|c|}
\hline Norma & Recomendação & FR \\
\hline \multirow{3}{*}{$\begin{array}{l}\text { SOGC - Female Sexual Health } \\
\text { Consensus Clinical Guidelines } \\
(2012)^{12}\end{array}$} & $\begin{array}{l}\text { Tratamento com androgénios pode ser efetivo em mulheres pós-menopáusicas com } \\
\text { DSH. }\end{array}$ & $A$ \\
\hline & $\begin{array}{l}\text { A relação da mulher com o/a companheiro/a e a sua saúde física e mental estão } \\
\text { mais correlacionadas com o desejo do que os } \mathrm{E} / \mathrm{T} \text {. }\end{array}$ & B \\
\hline & $\begin{array}{l}\text { São necessários mais estudos para conhecer os benefícios e os riscos a longo prazo } \\
\text { da terapêutica com androgénios no DSH na pós-menopausa. }\end{array}$ & $\mathrm{C}$ \\
\hline \multirow{2}{*}{$\begin{array}{l}\text { Androgen Therapy in Women: A } \\
\text { Reappraisal: An Endocrine Society } \\
\text { Clinical Practice Guideline (A Task } \\
\text { Force - the Endocrine Society, ACOG, } \\
\text { ASRM, ESE, IMS) }(2014)^{13}\end{array}$} & $\begin{array}{l}\text { Contra a utilização generalizada de T na DSF, exceto para o diagnóstico específico } \\
\text { de DSH na pós-menopausa. }\end{array}$ & A \\
\hline & $\begin{array}{l}\text { No DSH na pós-menopausa: } \\
\text { - O tratamento com T deve ser de 3-6 meses. } \\
\text { - Os níveis de T devem ser avaliados antes, e 3-6 semanas após o tratamento. } \\
\text { - Para tratamentos mais longos, avaliar os níveis de T a cada } 6 \text { meses. } \\
\text { - Suspender tratamento com T se ausência de resposta em } 6 \text { meses. }\end{array}$ & B \\
\hline $\begin{array}{l}\text { SOGC Clinical Practice Guideline - } \\
\text { Sexuality and Menopause - Managing } \\
\text { Menopause }(2014)^{14}\end{array}$ & $\begin{array}{l}\text { T TD } \uparrow \text { desejo sexual, excitação e frequência de relações sexuais satisfatórias e } \downarrow \\
\text { mal-estar em mulheres pós-menopáusicas. } \\
\text { Não existem produtos aprovados para esta indicação no Canadá. }\end{array}$ & A \\
\hline
\end{tabular}

ACOG: American College of Obstetricians and Gynecologists; ASRM: American Society for Reproductive Medicine; DSF: disfunção sexual feminina; DSH: desejo sexual hipoativo; E: estrogénios; ESSE: European Society of Endocrinology; FR: força de recomendação; IMS: International Menopause Society; SOGC: The Society of Obstetricians and Gynaecologists of Canada; T: testosterona; TD: transdérmica

As guidelines de 2012 da Society of Obstetricians and Gynaecologists of Canada (SOGC) - Female Sexual Health Consensus consideram que o tratamento com androgénios, quando apropriado, pode ser efetivo nas mulheres com DSH, mas que a afinidade com o/a companheiro/a e a saúde física e psicológica da mulher estão mais correlacionadas com o desejo sexual do que a testosterona, sendo necessários mais estudos de segurança a longo prazo. $^{12}$

Segundo a guideline da Endocrine Society (A Task For$c e$ - the Endocrine Society, American Congress of Obstetricians and Gynecologists - ACOG, American Society for Reproductive Medicine - ASRM, European Society of Endocrinology - ESE, International Menopause Society - IMS) de 2014, a utilização generalizada de testosterona na DSF não é recomendada, exceto para o diagnóstico específico de DSH na pós-menopausa. Nesta situação, é sugerido o tratamento de 3 - 6 meses com testosterona, e medição dos níveis desta hormona antes e 3 - 6 semanas após a terapêutica, de modo a avaliar os eventuais efeitos adversos do excesso de androgénios. Para tratamentos mais longos, recomenda avaliar os níveis de testosterona a cada seis meses. Propõe ainda a suspensão do tratamento, nas muIheres com ausência de resposta em seis meses, alegando não existirem dados suficientes que suportem a segurança e eficácia da testosterona a longo prazo. ${ }^{13}$

A guideline Sexuality and Menopause - Managing Menopause da SOGC de 2014, refere que a terapêutica transdérmica com testosterona aumenta o desejo sexual, a excitação e a frequência de relações sexuais satisfatórias e diminui o mal-estar em mulheres com menopausa natural ou cirúrgica, mas a aplicação desta recomendação é condicionada pelo facto de não existirem produtos aprovados para esta indicação no Canadá. ${ }^{14}$

\section{Revisão sistemática com meta-análise}

Achilli et a/15 realizaram uma RS com MA cujo objetivo foi rever e sumarizar a evidência relativa à eficácia e segurança da testosterona transdérmica em mulheres pós-menopáusicas (fisiológica ou cirúrgica) com DSH. As caraterísticas deste trabalho estão resumidas na Tabela 2.

O outcome primário foi definido como o número de episódios sexuais satisfatórios e os outcomes secundários foram atividade sexual, orgasmo e parâmetros do Profile of Female Sexual Function (PFSF) nomeadamente o desejo sexual. Verificou-se que, nas mulheres que receberam testosterona, houve mais episódios sexuais satisfatórios ( $p$ $<0,00001)$, mais atividade sexual $(p<0,0001)$, mais orgasmos $(p<0,00001)$ e aumento do desejo $(p<0,00001)$. Apresentaram também um aumento de eventos androgénicos adversos totais $(p=0,002)$, acne $(p=0,02)$ e crescimento do cabelo $(p=0,003)$. Não houve diferença entre os dois grupos no crescimento de pelo facial $(p=0,54)$, alopécia $(p=0,90)$, virilização da voz $(p=0,60)$, sintomas urinários, mastodinia, cefaleia, reação local ao sistema transdérmico, eventos adversos totais $(p=0,77)$ e severos $(p=$ $0,94)$, razões de descontinuação do estudo $(p=0,33)$ ou número de mulheres que completaram o estudo $(p=0,14)$.

Não foram estudados os efeitos a longo prazo do uso da testosterona transdérmica no cancro da mama, doença $\mathrm{CV}$, cognição, sintomas menopáusicos, alterações de humor, sensação de bem-estar ou densidade mineral óssea. Atribuímos um nível de evidência 1 a este estudo.

\section{Ensaios clínicos aleatorizados e controlados Caraterísticas dos estudos}

O resumo das caraterísticas dos estudos incluídos está sumarizado nas Tabelas 2 e 3.16-22 A duração do tratamento variou entre 8 semanas e 12 meses. As doses e a forma de administração variaram entre os estudos e foram avaliadas 
Tabela 2 - Resumo das caraterísticas da revisão sistemática com meta-análise e dos ensaios clínicos aleatorizados e controlados

\begin{tabular}{|c|c|c|c|c|}
\hline Estudo & População & Amostra & Grupo intervenção & Grupo controlo \\
\hline \multirow[t]{4}{*}{$\begin{array}{l}\text { RS com MA: } \\
\text { Achilli et al }(2017)^{15}\end{array}$} & $\begin{array}{l}\text { Menopausa } \\
\text { fisiológica ou } \\
\text { cirúrgica com DSH }\end{array}$ & $\begin{array}{l}7 \text { ECAC } \\
n=3035\end{array}$ & $\begin{array}{l}300 \mu g \text { T TD } \\
+\end{array}$ & $\begin{array}{l}\text { Placebo } \\
+\end{array}$ \\
\hline & & $\begin{array}{l}\text { Intervenção - } \\
1350 \\
\text { Controlo - } 1379\end{array}$ & $\begin{array}{l}\text { 1) } E(4 E C A C) \\
\text { 2) } P \text { e E (1 ECAC) }\end{array}$ & $\begin{array}{l}\text { 1) } E \text { (4 ECAC) } \\
\text { 2) } P \text { e } E(1 E C A C)\end{array}$ \\
\hline & & & 3) Sem E ou P (1 ECAC) & 3) Sem E ou P (1 ECAC) \\
\hline & & & 4) Com e sem E (1 ECAC) & 4) Com e sem E (1 ECAC) \\
\hline \multirow{2}{*}{$\begin{array}{l}\text { Warnock et al } \\
(2005)^{16}\end{array}$} & $25-65$ anos & $n=102$ & $1,25 \mathrm{mg} /$ dia $\mathrm{E}$ oral & $1,25 \mathrm{mg} /$ dia $\mathrm{E}$ oral \\
\hline & $\begin{array}{l}\text { Menopausa cirúrgica } \\
\text { com DSH sob E há } \\
3 \text { meses }\end{array}$ & $\begin{array}{l}\text { Intervenção - } 52 \\
\text { Controlo - } 50\end{array}$ & $\begin{array}{l}+ \\
2,5 \mathrm{mg} / \mathrm{dia} \text { MT oral }\end{array}$ & $\begin{array}{l}+ \\
\text { Placebo }\end{array}$ \\
\hline
\end{tabular}

Nathorst - Boos et al
$(2006)^{17}$

El-Hage et al $(2007)^{18}$

\section{$50-65$ anos \\ Menopausa \\ fisiológica com queixas $\downarrow$ libido sob THS}

$\mathrm{n}=53$

Grupo $1-27$

Grupo 2 - 26
Menopausa cirúrgica $\quad n=36$

com DSH sob E transdérmico
Grupo 1 - 18

Grupo 2 - 18

\section{GRUPO 1}

3 m.: 10 mg/dia T percutânea

$3 \mathrm{~m}$.: placebo

\section{GRUPO 2}

$3 \mathrm{~m}$.: placebo

3 m.: 10 mg/dia T percutânea

\section{GRUPO 1}

12 sem.: $10 \mathrm{mg} / \mathrm{dia}$ T creme

4 sem. sem tratamento

12 sem.: creme placebo

\section{GRUPO 2}

12 sem.: creme placebo

4 sem. sem tratamento

12 sem.: 10 mg/dia T creme

BISF-W: Brief Index of Sexual Function for Women; CSFQ-F-C: Changes in Sexual Functioning Questionnaire - Female Clinic Version; DSH: desejo sexual hipoativo; E: estrogénio; M: meses/mês; MA: meta-análise; MSIQ: Menopausal Sexual Interest Questionnaire; MT: metiltestosterona; NE: nível de evidência atribuído; P: progestagénio; PGWB: Psychological General Wellbeing - questionnaire; RS: revisão sistemática; Sem: semanas; T: testosterona; THS: terapêutica hormonal de substituição

mulheres com menopausa fisiológica e cirúrgica. As mulheres tinham entre 25 e 70 anos. As escalas e questionários para avaliação de função e desejo sexual também diferiram.

\section{Melhoria da função sexual}

Warnock et a/16 definiram como outcome primário a variação do valor médio da pontuação da subescala desejo/interesse sexual da Changes in Sexual Functioning Questionnaire - Female Clinic Version (CSFQ-F-C) após oito semanas de tratamento com testosterona. Como outcomes secundários consideraram as variações na pontuação média das escalas Menopausal Sexual Interest Questionnaire (MSIQ) e Women's Health Questionnaire (WHQ). A função sexual foi, assim, avaliada por duas escalas: a CSFQ-F-C, cuja pontuação total não mostrou melhoria significativa comparativamente com o grupo controlo ( $p=0,063$ ), pelo que o outcome primário não foi considerado cumprido, e a WHQ, que mostrou melhoria apenas no grupo tratado com estrogénio.

Nathorst-Boos et a/17 avaliaram a função sexual através do McCoy's Sex Scale Questionnaire (versão modificada), tendo havido uma melhoria estatisticamente significativa em vários parâmetros, comparativamente com o grupo que não recebeu testosterona, nomeadamente frequência de atividade sexual $(p<0,001)$, orgasmo $(p<0,001)$, excitação $(p<0,001)$ e prazer $(p<0,001)$. Foi também utilizado o questionário Psychological General Well-Being (PGWB), tendo-se verificado uma melhoria significativa do total e das subescalas ansiedade $(p<0,001)$ e bem-estar $(p=0,010)$ tanto antes como após o crossover, nos grupos aos quais foi administrada testosterona.

El-Hage et a/ ${ }^{18}$ definiram como outcome primário a melhoria na pontuação da sexualidade medida pelo questionário 


\section{Follow-up Melhoria da função sexual comparativamente} com grupo(s) não tratado(s) com T (valor $p$ )

Melhoria do desejo sexual

comparativamente com grupo(s) não tratado(s) com T (valor $p$ )

24 sem.

Melhoria dos episódios sexuais satisfatórios ( $p<0,00001)$, atividade sexual $(p<0,0001)$ e orgasmos $(p<0,00001)$

(exceto num ECAC, que durou $32 \mathrm{sem}$.)

\begin{tabular}{|c|c|}
\hline $8 \mathrm{sem}$. & $\begin{array}{l}\text { - CSFQ-F-C (pontuação total): } \\
\text { sem melhoria }(p=0,063) \\
\text { - CSFQ-F-C (pontuação subescala } \\
\text { excitabilidade/ereção): } \\
\text { melhoria }(p=0,009) \\
\text { - WHQ: } \\
\text { sem melhoria }\end{array}$ \\
\hline $\begin{array}{l}3 \mathrm{~m} . \\
+ \\
3 \mathrm{~m} . \\
\\
\text { (Total } 6 \mathrm{~m} .)\end{array}$ & $\begin{array}{l}\text { - McCoy's Sex Scale Questionnaire (versão } \\
\text { modificada): melhoria da frequência de ativida } \\
\text { sexual }(p<0,001) \text {, orgasmo }(p<0,001) \text {, excita } \\
\text { sexual }(p<0,001) \text { e prazer }(p<0,001)\end{array}$ \\
\hline $\begin{array}{l}12 \text { sem. } \\
+ \\
4 \text { sem. sem } \\
\text { tratamento } \\
+ \\
12 \text { sem. }\end{array}$ & $\begin{array}{l}\text { - BISF-W: melhoria do score total }(p=0,000) \text {, } \\
\text { frequência de relações sexuais }(p=0,039) \text { e } \\
\text { recetividade/iniciativa sexual }(p=0,000)\end{array}$ \\
\hline
\end{tabular}

\author{
- CSFQ-F-C (pontuação subescala desejo/ \\ interesse sexual): sem melhoria $(p=0,644)$
}

- MSIQ: com melhoria $(p=0,012)$

\author{
- McCoy's Sex Scale Questionnaire \\ (versão modificada): melhoria dos \\ pensamentos sexuais/fantasias $(p<0,001) \mathrm{e}$ \\ interesse sexual $(p<0,01)$
}

- BISF-W: melhoria do desejo sexual $(p=0,024)$
Brief Index of Sexual Function for Women (BISF-W). Os autores observaram um aumento significativo do score total $(p=0,000)$, da frequência de relações sexuais $(p=0,039)$ e da recetividade/ iniciativa sexual $(p=0,000)$ comparativamente com o grupo que não recebeu testosterona.

de Paula et al ${ }^{19}$ utilizaram o questionário McCoy's Sex Scale Questionnaire (versão modificada) para avaliar a função sexual, verificando-se nos grupos que receberam testosterona, uma melhoria estatisticamente significativa da satisfação sexual após 2 e 4 meses de tratamento $(p<$ 0,05).

Penteado et $a^{20}$ avaliaram a energia sexual, conceito que inclui sonhos e fantasias sexuais, sensação ou tensão genital e apetite sexual, utilizando a Sexual Energy Change Scale, a capacidade de atingir orgasmo e outros parâmetros sexuais (masturbação, frequência de orgasmo, relação sexual vaginal, desejo/excitação sexual e secura vaginal). No grupo que recebeu testosterona verificou-se melhoria da perceção subjetiva do nível de energia sexual $(p=0,021)$, sendo significativa a diferença relativamente aos relatos de 'grande melhoria' $(p=0,038)$ e 'resolução completa dos problemas relacionados com o nível energia sexual' $(p=0,028)$. Não se observaram diferenças estatisticamente significativas na capacidade de atingir orgasmo após adição de testosterona $(p=0,553)$. Relativamente aos restantes parâmetros, verificou-se em ambos os grupos, comparativamente à baseline, um aumento significativo na frequência de orgasmos $(p<0,001)$ e de relações sexuais vaginais $(p<0,001)$, bem como uma melhoria do desejo/ excitação sexual $(p<0,001)$ e sintomas de secura vaginal $(p<0,01)$.

Fernandes et $a^{21}$ avaliaram a função sexual através do questionário Female Sexual Function Index (FSFI), onde verificaram uma melhoria estatisticamente significativa de vários parâmetros nomeadamente no score total ( $p=$ $0,003)$, lubrificação $(p=0,032)$, satisfação $(p=0,022)$ e dor $(p=0,013)$ comparativamente com o grupo controlo (grupo que recebeu lubrificante). $\mathrm{O}$ tratamento com testosterona 
Tabela 3 - Resumo das caraterísticas dos ensaios clínicos aleatorizados e controlados (continuação)

\begin{tabular}{|c|c|c|c|c|}
\hline Estudo & População & Amostra & Grupo intervenção & Grupo controlo \\
\hline $\begin{array}{l}\text { de Paula et al } \\
(2007)^{19}\end{array}$ & $\begin{array}{l}49 \text { - } 63 \text { anos } \\
\text { Menopausa fisiológica } \\
\text { com queixas de } \\
\text { disfunção sexual sob } \\
\text { THS há } \geq 1 \text { ano }\end{array}$ & $\begin{array}{l}n=85 \\
4 \text { Grupos de } \\
\text { Tratamento }\end{array}$ & \multicolumn{2}{|c|}{$\begin{array}{l}\text { GRUPO I: THS + placebo } \\
\text { GRUPO II: THS + 2,5 mg/dia MT oral } \\
\text { GRUPO III: THS + placebo } 2 \mathrm{~m} \text {; d depois THS + } 2,5 \mathrm{mg} / \text { dia MT } \\
\text { oral } 2 \mathrm{~m} \text {. } \\
\text { GRUPO IV: THS + } 2,5 \mathrm{mg} / \text { dia MT oral } 2 \mathrm{~m} \text {.; depois THS + } \\
\text { placebo } 2 \mathrm{~m} \text {. }\end{array}$} \\
\hline $\begin{array}{l}\text { Penteado et al } \\
(2008)^{20}\end{array}$ & $\begin{array}{l}42 \text { - } 60 \text { anos } \\
\text { Menopausa fisiológica } \\
\text { com queixas sexuais } \\
\text { sem tratamento } \\
\text { hormonal prévio }\end{array}$ & $\begin{array}{l}\mathrm{n}=60 \\
\text { Intervenção } \\
-31 \\
\text { Controlo - } 29\end{array}$ & $\begin{array}{l}0,625 \mathrm{mg} / \mathrm{dia} \text { E oral } \\
+ \\
2,5 \mathrm{mg} / \mathrm{dia} \mathrm{P} \text { oral } \\
+ \\
2 \mathrm{mg} / \mathrm{dia} \mathrm{MT} \text { oral }\end{array}$ & $\begin{array}{l}0,625 \mathrm{mg} / \mathrm{d} \text { E oral } \\
+ \\
2,5 \mathrm{mg} / \mathrm{d} \mathrm{P} \text { oral } \\
+ \\
\text { placebo oral }\end{array}$ \\
\hline $\begin{array}{l}\text { Fernandes et al } \\
(2014)^{21}\end{array}$ & $\begin{array}{l}40 \text { - } 70 \text { anos } \\
\text { Menopausa fisiológica } \\
\text { com queixas de atrofia } \\
\text { vaginal sem tratamento } \\
\text { hormonal prévio }\end{array}$ & $\begin{array}{l}\mathrm{n}=80 \\
\text { Divididos por } 4 \\
\text { grupos, cada } \\
\text { um com } \mathrm{n}=20\end{array}$ & $\begin{array}{l}\text { - Creme vaginal com ác. } \\
\text { poliacrílico } \\
\text { vs } \\
\text { - Creme vaginal com PT } \\
\text { vs } \\
\text { - Creme vaginal com E }\end{array}$ & Creme vaginal com glicerina \\
\hline $\begin{array}{l}\text { Tungmunsakulchai } \\
\text { et al }(2015)^{22}\end{array}$ & $\begin{array}{l}40 \text { - } 60 \text { anos } \\
\text { Menopausa fisiológica } \\
\text { ou cirúrgica com } \\
\text { disfunção sexual sem } \\
\text { tratamento hormonal } \\
\text { prévio }\end{array}$ & $\begin{array}{l}\mathrm{n}=70 \\
\text { Intervenção } \\
-35 \\
\text { Controlo - } 35\end{array}$ & $\begin{array}{l}40 \mathrm{mg} 2 \times \text { /sem. UT oral } \\
+ \\
1 \mathrm{mg} / \mathrm{d} \text { E oral }\end{array}$ & $\begin{array}{l}40 \mathrm{mg} 2 \times \text { /sem. placebo oral } \\
+ \\
1 \mathrm{mg} / \mathrm{d} \text { E oral }\end{array}$ \\
\hline
\end{tabular}

DSH: desejo sexual hipoativo; E: estrogénio; ECAC: ensaio clínico aleatorizado e controlado; FSFI: Female Sexual Function Index; G: grupo; M: meses/mês; MT: metiltestosterona; NE: nível de evidência atribuído; P: progestagénio; PT: propionato de testosterona; Sem: semanas; T: testosterona; TD: transdérmica, THS: tterapêutica hormonal de substituição; UT: undecanoato de testosterona

mostrou o melhor resultado, comparando com os outros tratamentos, na melhoria da função sexual.

Tungmunsakulchai et $a^{R 2}$ realizaram um estudo onde definiram como outcome primário a melhoria na pontuação no questionário FSFI. Os autores verificaram uma melhoria estatisticamente significativa na pontuação total $(p=0,04)$ e no parâmetro da excitabilidade $(p=0,02)$ do FSFI no grupo sujeito a terapêutica com testosterona, comparativamente com o grupo controlo.

\section{Melhoria do desejo sexual}

Seis dos sete estudos incluídos ${ }^{16-21}$ mostraram melhoria do desejo sexual nos grupos tratados com testosterona, independentemente da via de administração, dose ou duração do tratamento.

Warnock et a ${ }^{16}$ avaliaram a melhoria do desejo sexual através de dois questionários, o MSIQ, que mostrou melhoria significativa comparativamente com grupo controlo $(p=$ 0,012), e o CSFQ-F-C (pontuação da subescala desejo/interesse sexual, correspondente ao outcome primário), que não mostrou melhoria significativa $(p=0,644)$.

Nathorst-Boos et a ${ }^{17}$ avaliaram o desejo sexual através do McCoy's Sex Scale Questionnaire (versão modificada), tendo observado uma melhoria estatisticamente significati- va comparativamente com o grupo que não recebeu testosterona (antes e após o crossover) nos parâmetros pensamentos sexuais/fantasias $(p<0,001)$ e interesse sexual $(p$ $<0,001)$.

El-Hage et a ${ }^{18}$ avaliaram a melhoria do desejo sexual através do questionário Brief Index of Sexual Function for Women (BISF-W), com melhoria significativa do parâmetro relativo ao desejo sexual $(p=0,024)$, comparativamente com o grupo que não recebeu testosterona.

de Paula et al, ${ }^{19}$ utilizando o questionário McCoy's Sex Scale Questionnaire (versão modificada), verificaram uma melhoria estatisticamente significativa do desejo sexual após 2 e 4 meses de tratamento com testosterona $(p<0,01$ $-0,05$ e $p<0,05$ ), comparativamente ao grupo que recebeu placebo.

Os resultados observados por Penteado et al,,$^{20}$ no que diz respeito ao desejo sexual, já foram descritos acima, tendo-se observado, no grupo que recebeu testosterona, uma melhoria estatisticamente significativa da perceção subjetiva de energia sexual $(p=0,021)$.

Fernandes et $a^{R 1}$ avaliaram a melhoria do desejo sexual através do questionário FSFI, onde houve uma melhoria estatisticamente significativa do parâmetro relativo ao desejo sexual $(p<0,001)$ comparativamente com o lubrificante. 


\begin{tabular}{ll} 
Follow-up & $\begin{array}{l}\text { Melhoria da função sexual comparativamente } \\
\text { com grupo(s) não tratados com testosterona } \\
(\text { valor } p)\end{array}$ \\
\hline
\end{tabular}

$\begin{array}{lc}2 \text { m. (T1) } & \text { - McCoy's Sex Scale Questionnaire (versão } \\ + & \text { modificada): melhoria da satisfação sexual } \\ 2 \text { m. (T2) } & \text { T1: GII }(p<0,05) \text { e GIV }(p<0,05) \\ & \text { T2: GII }(p<0,05) \text { e GIII }(p<0,05)\end{array}$

(Total: 4 m.)
Melhoria do desejo sexual comparativamente com grupo(s) não tratados com testosterona (valor $p$ )

- McCoy's Sex Scale Questionnaire (versão modificada): melhoria do desejo sexual

T1: GII $(p<0,05)$ e GIV $(p<0,01)$

T2: GII $(p<0,05)$ e GIII $(p<0,05)$

$12 \mathrm{~m}$.

- Sexual Energy Change Scale: melhoria da energia sexual global $(p=0,021)$
- Sexual Energy Change Scale: melhoria da energia sexual global $(p=0,021)$
1

- Orgasmic Capacity: ausência de melhoria $(p=0,553)$

12 sem.

- FSFI: melhoria na pontuação global $(p=0,003)$, lubrificação $(p=0,032)$, satisfação $(p=0,022)$ e $\operatorname{dor}(p=0,013)$ na excitabilidade $(p=0,02)$

O único estudo que não mostrou melhoria do desejo sexual após tratamento com testosterona foi o trabalho realizado por Tungmunsakulchai et al. ${ }^{22}$ Os autores utilizaram o questionário $\mathrm{FSFI}$ e no parâmetro do desejo não houve melhoria estatisticamente significativa $(p=0,99)$ comparativamente com o grupo controlo.

\section{Qualidade dos estudos incluídos}

A seis dos sete estudos incluídos ${ }^{16-20,22}$ foi atribuído um nível de evidência 1 , uma vez que foram estudos com evidência orientada para o doente, aleatorizados e controlados, com uma amostra adequada e duplo-cegos. Ao estudo realizado por Fernandes et $a^{21}$ atribuímos um nível de evidência 2, por não ter sido um estudo verdadeiramente duplo cego.

\section{Tipo de intervenção}

A posologia utilizada, o tipo de molécula de testosterona e o grupo comparativo diferiu bastante entre os diferentes estudos.

$\mathrm{Na}$ RS com MA de Achilli et al, ${ }^{15}$ o grupo de intervenção recebeu $300 \mu \mathrm{g}$ de testosterona ( $\mathrm{T}$ ) transdérmica e o grupo controlo placebo. Adicionalmente, as mulheres receberam também estrogénio $(E)$, progesterona $(P)$, ambas ou nenhuma destas hormonas. $O$ tratamento durou 24 semanas, exceto num ECAC que durou 32 semanas.
Com Warnock et al, ${ }^{16}$ o grupo de intervenção recebeu $\mathrm{E}+2,5 \mathrm{mg} /$ dia de metiltestosterona (MT), orais, e o grupo controlo $\mathrm{E}+$ placebo, orais. O tratamento durou oito semanas.

No estudo de Nathorst-Boos et al, ${ }^{17}$ o grupo 1 recebeu 10 mg/dia de T percutânea ( 3 meses) e placebo (nos 3 meses seguintes); o grupo 2 recebeu placebo (3 meses) e 10 $\mathrm{mg} / \mathrm{dia}$ de $\mathrm{T}$ percutânea (nos 3 meses seguintes).

No ECAC de El-Hage et $a l,^{18}$ o primeiro grupo recebeu $10 \mathrm{mg} /$ dia de T creme (12 semanas) + ausência de tratamento (4 semanas) + creme placebo (12 semanas). O segundo recebeu creme placebo (12 semanas) + ausência de tratamento (4 semanas) $+10 \mathrm{mg} / \mathrm{dia}$ de $\mathrm{T}$ creme $(12$ semanas).

No ECAC de de Paula et al, ${ }^{19}$ foram formados quatro grupos de tratamento: grupo 1 (terapêutica hormonal de substituição (THS)) + placebo - 4 meses), grupo 2 (THS + $2,5 \mathrm{mg} / \mathrm{dia}$ de MT oral -4 meses), grupo 3 (THS + placebo -2 meses, depois THS $+2,5 \mathrm{mg} / \mathrm{dia}$ de MT oral -2 meses) e grupo 4 (THS $+2,5 \mathrm{mg} / \mathrm{dia}$ de MT oral -2 meses, depois THS + placebo -2 meses).

Com Penteado et al, ${ }^{20}$ o grupo de intervenção recebeu $\mathrm{E}+\mathrm{P}+2 \mathrm{mg} / \mathrm{dia}$ de MT, orais, e o grupo de controlo recebeu $\mathrm{E}+\mathrm{P}+$ placebo, orais. O tratamento durou 12 meses.

No ECAC de Fernandes et a ${ }^{21}$ participaram quatro grupos (todos receberam o tratamento sob a forma de creme 
vaginal): o primeiro recebeu ácido poliacrílico, o segundo propionato de $\mathrm{T}$, o terceiro $\mathrm{E}$, e o quarto placebo (lubrificante de glicerina). O tratamento durou 12 semanas.

No último estudo, de Tungmunsakulchai et al, ${ }^{22}$ o grupo de intervenção recebeu $40 \mathrm{mg}$ de undecanoato de T (2 x/ semana) $+E$, orais, e o segundo grupo recebeu placebo (2 $x$ / semana) $+E$, orais. A duração foi de oito semanas.

\section{Efeitos adversos}

Todos os sete estudos incluídos ${ }^{16-22}$ avaliaram os efeitos adversos da terapêutica. Verificou-se um aumento da incidência da acne apenas em dois estudos, ${ }^{16,20}$ sendo que em ambos foi administrada testosterona por via oral. Warnock et a ${ }^{16}$ administraram $2,5 \mathrm{mg} / \mathrm{dia}$ de metiltestosterona oral durante oito semanas e Penteado et $a^{20}$ administraram 2 $\mathrm{mg}$ de testosterona oral durante um período de 12 meses. Warnock et $a{ }^{16}$ registaram também como efeitos adversos aumento de peso, ansiedade e vaginite. Nenhum dos sete estudos mostrou efeitos adversos considerados graves. ${ }^{16-22}$

\section{Alterações laboratoriais}

Cinco dos sete estudos incluídos avaliaram a existência de alterações laboratoriais, nomeadamente no perfil lipídico e enzimas hepáticas. ${ }^{16-19,22}$ Destes, apenas Warnock et $a l,{ }^{16}$ que utilizaram testosterona oral, verificaram alterações a nível do perfil lipídico com uma redução estatisticamente significativa, comparativamente com o grupo controlo, do colesterol HDL $(p<0,001)$ e colesterol total $(p<0,001)$.

\section{DISCUSSÃO}

Globalmente, as NOC, a RS com MA e os ECAC incluídos nesta revisão evidenciaram que o uso de testosterona em mulheres em pós-menopausa é eficaz, a curto prazo, na melhoria da função sexual.

Consideramos como ponto forte desta revisão a pesquisa criteriosa e a avaliação de elegibilidade dos estudos. Poderão constituir limitações à interpretação dos resultados o facto de fatores individuais, relacionais e culturais influenciarem a perceção e vivência da sexualidade, dado que os estudos avaliaram mulheres de diferentes regiões geográficas (Brasil, Tailândia, Suécia, entre outras). Para além disto, houve inclusão, nos diferentes estudos, de muIheres com tipos distintos de disfunção e foram utilizados diferentes critérios para a definição de diminuição do desejo sexual e diferentes instrumentos para avaliação dos outcomes, devendo neste ponto referir-se que o questionário CSFQ-F-C, utilizado por Warnock et a/ ${ }^{16}$ para avaliação do desejo sexual, não se encontra validado para a população em estudo, considerando os autores que utilizar esta escala poderá ter sido um erro. Outra limitação desta revisão prende-se com o facto de três dos ECAC incluídos terem sido patrocinados por laboratórios responsáveis pela comercialização dos produtos administrados. ${ }^{17,18,20}$

Não existe consenso no que diz respeito ao tipo de molécula de testosterona e à sua posologia (dose, via de administração ou duração do tratamento), dado que estes parâmetros foram diferentes nos diversos estudos analisa- dos. Relativamente às vias de administração, verificámos que nos seis ECAC em que a testosterona foi administrada em associação com estrogénios (com ou sem progesterona), foram utilizadas as vias oral e transdérmica. ${ }^{16-20,22}$ No único ECAC que avaliou os efeitos da testosterona em monoterapia, esta foi administrada por via vaginal. ${ }^{21}$

No que respeita à associação de testosterona com outras terapêuticas (como estrogénios), inferiu-se que todos os benefícios observados resultaram da ação da testosterona. A importância desta associação prende-se com o facto da diminuição do interesse sexual poder ser confundida com sintomas de deficiência de estrogénios. ${ }^{16,23}$

Todos os ECAC e a RS com MA avaliaram os efeitos adversos da terapêutica, verificando-se um aumento da incidência de acne apenas na RS com MA ${ }^{15}$ que utilizou testosterona transdérmica, e em dois ECAC, ${ }^{16,20}$ que utilizaram testosterona oral. Para além disto, nenhum dos estudos evidenciou alterações no perfil lipídico, enzimas hepáticas, IMC ou efeitos adversos graves, à exceção de Warnock et al, ${ }^{16}$ que utilizaram $2 \mathrm{mg}$ de testosterona oral e verificaram um aumento do peso e alterações a nível do perfil lipídico, com uma redução estatisticamente significativa do colesterol HDL e colesterol total, comparativamente com o grupo controlo, podendo sugerir um perfil mais desfavorável de efeitos adversos com a formulação oral. É importante referir que estes resultados poderão estar relacionados não só com a reduzida dimensão das amostras dos ECAC mas principalmente com a curta duração do follow-up (entre 2 e 12 meses), o que impossibilitou a avaliação da eficácia terapêutica da testosterona e dos seus efeitos adversos a longo prazo.

Os resultados obtidos por esta revisão são coincidentes com a maioria dos resultados encontrados na literatura, sobretudo no que respeita ao efeito benéfico da testosterona na função sexual. Uma revisão da Cochrane publicada em 2005 e conduzida por Somboonporn ${ }^{24}$ mostrou que associar testosterona em qualquer formulação a terapêutica hormonal, em mulheres na pós-menopausa (fisiológica ou cirúrgica), tem um efeito benéfico na função sexual. Foi associada, tal como na revisão que apresentamos, a um aumento da incidência da acne e a uma redução do colesterol HDL. Contudo, houve também um aumento do pelo facial, que não foi demonstrado na nossa revisão. Esta revisão concluiu que não existe evidência suficiente para determinar os seus efeitos a longo prazo.

A revisão da literatura conduzida por Hubayter et $a l,{ }^{2}$ publicada em 2008, mostrou que os efeitos adversos são bem tolerados e reversíveis e concluiu que a terapêutica com testosterona deve ser considerada em mulheres com DSH. Esta revisão também concluiu que as formulações transdérmicas são preferíveis às outras formulações.

Kingsberg et a ${ }^{25}$ conduziram uma revisão da literatura, publicada em 2008, que mostrou que as mulheres em menopausa cirúrgica que receberam testosterona melhoraram significativamente a sua função sexual comparativamente com o placebo, com um perfil de segurança favorável. Referem, tal como a nossa revisão, que são necessários 
novos estudos de longa duração para determinar a segurança a longo prazo da testosterona, sobretudo no que respeita aos eventos cardiovasculares e cancro da mama. Ainda em relação à segurança da testosterona, Braunstein ${ }^{26}$ concluiu que exceto para o hirsutismo e acne, a administração terapêutica da testosterona em doses fisiológicas é segura.

Schover ${ }^{27}$ contudo, encontrou resultados divergentes após revisão da literatura em 2008, mostrando haver apenas um benefício modesto da terapêutica com testosterona e referindo uma possível associação com o cancro da mama com base em dados epidemiológicos.

Está a decorrer um estudo aleatorizado e controlado para avaliar a segurança CV e risco de cancro da mama com a utilização da testosterona em gel no tratamento a longo prazo da DSF, em especial o DSH. Estão incluídas mulheres pós-menopáusicas com DSH e aumento do risco $\mathrm{CV}$, com um período de follow-up de cinco anos. Este estudo poderá ajudar a definir o perfil de segurança a longo prazo da testosterona em baixa dose. ${ }^{28}$

Em relação à avaliação dos resultados, o desejo e a excitação sexual foram apresentados de forma independente, uma vez que o nosso objetivo era avaliar a eficácia da testosterona na disfunção sexual, em particular na melhoria do desejo, e que todos os estudos incluídos separaram os dois conceitos.

\section{CONCLUSÃO}

Em relação aos benefícios da terapêutica com testosterona, a RS com MA e seis dos sete ECAC incluídos ${ }^{16-21}$ mostraram melhoria do desejo sexual nos grupos tratados com testosterona, independentemente da via de administração (oral, transdérmica ou vaginal), dose ou duração do tratamento. Assim, de acordo com a evidência disponível, podemos concluir que a terapêutica com testosterona em monoterapia ou associada a outras hormonas, tem benefício, a curto prazo, na melhoria das queixas de disfunção sexual em mulheres na pós-menopausa, em particular na melhoria do desejo sexual (atribuímos Força de Recomendação A).

Em relação aos efeitos adversos verificou-se um aumento da incidência de acne apenas na RS com MA ${ }^{15}$ (foi utilizada testosterona transdérmica), e em dois ECAC $^{16,20}$ (utilizaram testosterona oral). Para além disto, apenas Warnock et $a /^{15}$ (utilizaram testosterona oral) evidenciaram um aumento do peso e alterações a nível do perfil lipídico, o que pode sugerir um perfil mais desfavorável com a formulação oral.

Estas conclusões são suportadas por um elevado número de estudos, a maioria de boa qualidade, com resultados consistentes e orientados para o paciente. Contudo, são necessários estudos com amostras de maior dimensão e período de follow-up mais longo, de modo a avaliar a sua efetividade e segurança a longo prazo.

\section{CONFLITOS DE INTERESSE}

Os autores declaram não terem qualquer conflito de interesse relativamente ao presente artigo.

\section{FONTES DE FINANCIAMENTO}

A realização deste artigo ocorreu sem qualquer financiamento exterior.

\section{REFERÊNCIAS}

1. Dynamed: Female sexual dysfunction. [consultado 2017 mar 9]. Disponível em http://web.ebscohost.com/dynamed/ detail?sid=6cb9c9e7-5d3f-475a-8b72-46ffe9d0342b\%40sessionmgr 12 0 \&vid=3\&hid=125\&bdata $=$ Jmxhbmc9cHQtYnlmc2I0ZT1 keW5hbWVkL WxpdmUmc2NvcGU9c2I0ZQ\%3d\%3d\#db=dme\&AN=116816.

2. Hubayter Z, Simon JA. Testosterone therapy for sexual dysfunction in postmenopausal women. Climacteric. 2008;11:181-91.

3. Pluchino N, Carmignani A, Cubeddu A, Santoro A, Cela V, Alcalà TE. Androgen therapy in women: for whom and when. Arch Gynecol Obstet. 2013;288:731-7.

4. Kingsberg AS, Rezaee RL. Hypoactive sexual desire in women. Menopause. 2013;3:1284-300.

5. Faubion SS, Rullo JE. Sexual dysfunction in women: a practical approach. Am Fam Physician. 2015;92:281-8.

6. American Psychiatric Association. Diagnostic and statistical manual of mental disorders - text revision, DSM IV-TR. $4^{\text {th }}$ ed. Washington: American Psychiatric Association; 2000.

7. American Psychiatric Association. Diagnostic and statistical manual of mental disorders, DSM-5. $5^{\text {th }}$ ed. Washington: American Psychiatric Association; 2013.

8. Shifren JL. Testosterone for midlife women: the hormone of desire? Menopause. 2015;22:1147-9.

9. Shifren JL, Braunstein GD, Simon JA, Casson PR, Buster JE, Redmond $\mathrm{GP}$, et al. Transdermal testosterone treatment in women with impaired sexual function after oophorectomy. N Engl J Med. 2000;343:682-8.

10. Infarmed. Prontuário terapêutico. [consultado 2018 fev 22]. Disponível em http://app10.infarmed.pt/prontuario/framepesactivos.php?palavra=t estosterona\& $\mathrm{x}=0 \& \mathrm{y}=0 \& \mathrm{rb} 1=0$.

11. Ebell MH, Siwek J, Weiss BD, Woolf SH, Susman J, Ewigman B, et al. Strength of recommendation taxonomy (SORT): a patient-centered approach to grading evidence in the medical literature. Am Fam

Physician. 2004;69:548-56

12. Lamont J, Bajzak K, Bouchard C, Burnett M, Byers S, Cohen T, et al. Female Sexual Health Consensus Clinical Guidelines. J Obstet Gynaecol Can. 2012;34:S48-53.

13. Wierman M, Wiebke A, Basson R, Davis S, Miller K, Murad M, et al. Androgen therapy in women: a reappraisal: an endocrine society clinical practice guideline. J Clin Endocrinol Metab. 2014;99:3489-510.

14. Reid R, Abramson BL, Blake J, Desindes S, Dodin S, Johnston S, et al. Managing menopause. J Obstet Gynaecol Can. 2014;36:S59-73.

15. Achilli C, Pundir J, Ramanathan P, Sabatini L, Hamoda H, Panay N. Efficacy and safety of transdermal testosterone in postmenopausal women with hypoactive sexual desire disorder: a systematic review and meta-analysis. Fertil Steril. 2017;107:475-82.

16. Warnock JK, Swanson SG, Borel RW, Zipfel LM, Brennan JJ. Combined esterified estrogens and methyltestosterone versus esterified estrogens alone in the treatment of loss of sexual interest in surgically menopausal women. Menopause. 2005;12:374-84.

17. Nathorst-Boos J, Floter A, Jarkander-Rolff M, Carlstrom K, Schoultz B. Treatment with percutanous testosterone gel in postmenopausal women with decreased libido-effects on sexuality and psychological general well-being. Maturitas. 2006;53:11-8.

18. El-Hage G, Eden JA, Manga RZ. A double-blind, randomized, placebocontrolled trial of the effect of testosterone cream on the sexual motivation of menopausal hysterectomized women with hypoactive sexual desire disorder. Climacteric. 2007;10:335-43.

19. de Paula FJ, Soares JM Jr, Haidar MA, de Lima GR, Baracat EC. The benefits of androgens combined with hormone replacement therapy regarding to patients with postmenopausal sexual symptoms. Maturitas. 2007;56:69-77.

20. Penteado SR, Fonseca AM, Bagnoli VR, Abdo CH, Junior JM, Baracat EC. Effects of the addition of methyltestosterone to combined hormone 
therapy with estrogens and progestogens on sexual energy and on orgasm in postmenopausal women. Climacteric. 2008;11:17-25.

21. Fernandes T, Costa-Paiva LH, Pinto-Neto AM. Efficacy of vaginally applied estrogen, testosterone, or polyacrylic acid on sexual function in postmenopausal women: a randomized controlled trial. J Sex Med. 2014;11:1262-70.

22. Tungmunsakulchai $R$, Chaikittisilpa $S$, Snabboon T, Panyakhamlerd $\mathrm{K}$, Jaisamrarn U, Taechakraichana N. Effectiveness of a low dose testosterone undecanoate to improve sexual function in postmenopausal women. BMC Women's Health. 2015;15:113.

23. Sociedade Portuguesa de Ginecologia, Sociedade Portuguesa de Menopausa. Consensos \& estratégias para a saúde da mulher na pósmenopausa [e-book]. 2004. [consultado 2017 mar 13]. Disponível em: http://www.spginecologia.pt/consensos.html.
24. Somboonporn W, Bell RJ, Davis SR. Testosterone for peri and postmenopausal women. Cochrane Database Syst Rev. 2005:CD004509.

25. Kingsberg SA, Simon JA, Goldstein I. The current outlook for testosterone in the management of hypoactive sexual desire disorderin postmenopausal women. J Sex Med. 2008;5:182-93.

26. Braunstein GD. Safety of testosterone treatment in postmenopausal women. Fertil Steril. 2007;88:1-17.

27. Schover LR. Androgen therapy for loss of desire in women: is the benefit worth the breast cancer risk? Fertil Steril. 2008;90:129-40.

28. White WB, Grady D, Giudice LC, Berry SM, Zborowski J, Snabes MC. A cardiovascular safety study of LibiGel (testosterone gel) in postmenopausal women with elevated cardiovascular risk and hypoactive sexual desire disorder. Am Heart J. 2012;163:27-32. 